\title{
Evidence of Spin-Orbital Angular Momentum Interactions in Relativistic Heavy-Ion Collisions
}

\author{
S. Acharya et al. \\ (The ALICE Collaboration)
}

(Received 21 November 2019; revised 25 February 2020; accepted 27 May 2020; published 30 June 2020)

\begin{abstract}
The first evidence of spin alignment of vector mesons $\left(K^{* 0}\right.$ and $\left.\phi\right)$ in heavy-ion collisions at the Large Hadron Collider (LHC) is reported. The spin density matrix element $\rho_{00}$ is measured at midrapidity $(|y|<0.5)$ in $\mathrm{Pb}-\mathrm{Pb}$ collisions at a center-of-mass energy $\left(\sqrt{s_{\mathrm{NN}}}\right)$ of $2.76 \mathrm{TeV}$ with the ALICE detector. $\rho_{00}$ values are found to be less than $1 / 3(1 / 3$ implies no spin alignment) at low transverse momentum $\left(p_{T}<2 \mathrm{GeV} / c\right)$ for $K^{* 0}$ and $\phi$ at a level of $3 \sigma$ and $2 \sigma$, respectively. No significant spin alignment is observed for the $K_{S}^{0}$ meson ( $\operatorname{spin}=0$ ) in $\mathrm{Pb}-\mathrm{Pb}$ collisions and for the vector mesons in $p p$ collisions. The measured spin alignment is unexpectedly large but qualitatively consistent with the expectation from models which attribute it to a polarization of quarks in the presence of angular momentum in heavy-ion collisions and a subsequent hadronization by the process of recombination.
\end{abstract}

DOI: 10.1103/PhysRevLett.125.012301

Ultrarelativistic heavy-ion collisions create a system of deconfined quarks and gluons, called the quark-gluon plasma (QGP) [1-3] and provide the opportunity to study its properties. In collisions with nonzero impact parameter, a large angular momentum of $O\left(10^{7}\right) \hbar$ [4] and magnetic field of $O\left(10^{14}\right) \mathrm{T}$ [5] are also expected. While the magnetic field is short lived (a few $\mathrm{fm} / c$ ), the angular momentum is conserved and could affect the system throughout its evolution. Experimental observables like correlations in azimuthal angle [6,7] could be used to study the influence of these initial conditions on the properties and the dynamical evolution of the QGP and its subsequent hadronization.

Spin-orbit interactions have wide observable consequences in several branches of physics [8-10]. In the presence of a large angular momentum, the spin-orbit coupling of quantum chromodynamics (QCD) could lead to a polarization of quarks followed by a net-polarization of vector mesons $\left(K^{* 0}\right.$ and $\left.\phi\right)$ [11-15] along the direction of the angular momentum.

The spin state of a vector meson is described by a $3 \times 3$ Hermitian spin-density matrix [15]. Its trace is 1 and $\rho_{11}$ and $\rho_{-1-1}$ cannot be measured separately in two-body decays to pseudoscalar mesons. Consequently, there is only one independent diagonal element, $\rho_{00}$. The elements of the spin-density matrix can be studied by measuring the

\footnotetext{
*Full author list given at the end of the article.

Published by the American Physical Society under the terms of the Creative Commons Attribution 4.0 International license. Further distribution of this work must maintain attribution to the author(s) and the published article's title, journal citation, and DOI.
}

angular distributions of the decay products of vector mesons with respect to a quantization axis. Here two different quantization axes are used: (i) a vector perpendicular to the production plane (PP) of the vector meson and (ii) the normal to the reaction plane (RP) of the system. The PP is defined by the flight direction of the vector meson and the beam direction.

The spin-density matrix element $\rho_{00}$ is determined from the distribution of the angle $\theta^{*}$ between the kaon decay daughter and the quantization axis in the decay rest frame [16,17],

$$
\frac{d N}{d \cos \theta^{*}} \propto\left[1-\rho_{00}+\cos ^{2} \theta^{*}\left(3 \rho_{00}-1\right)\right] .
$$

$\rho_{00}$ is $1 / 3$ in the absence of spin alignment and the angular distribution in Eq. (1) is uniform. The experimental signature of spin alignment is a nonuniform angular distribution $\left(\rho_{00} \neq 1 / 3\right)$.

The direction of the angular momentum in noncentral heavy-ion collisions is perpendicular to the reaction plane (subtended by the beam axis and impact parameter) [12]. The spin-orbit interaction is expected to lead to spin alignment with respect to the RP. The reaction plane orientation cannot be measured directly, but is estimated from the final state distributions of particles. This experimentally measured plane is called the event plane (EP) [18]. The deviation of the EP with respect to the RP is corrected using the EP resolution $(R)$ and observed $\rho_{00}^{\text {obs }}[19]$,

$$
\rho_{00}=\frac{1}{3}+\left(\rho_{00}^{\mathrm{obs}}-\frac{1}{3}\right) \frac{4}{1+3 R} .
$$


There are specific qualitative predictions for the spin alignment effect [13]: (a) $\rho_{00}>1 / 3$ if the hadronization of a polarized parton proceeds via fragmentation and less than $1 / 3$ for hadronization via recombination, (b) $\rho_{00}$ is expected to have a smaller deviation from $1 / 3$ for both central (impact parameter $\lesssim 3 \mathrm{fm}$ ) and peripheral (impact parameter $\gtrsim 11 \mathrm{fm}$ ) heavy-ion collisions, and a maximum deviation for mid-central collisions, where the angular momentum is also maximal, (c) the $\rho_{00}$ value is expected to have maximum deviation from $1 / 3$ at low $p_{T}$ and reach the value of $1 / 3$ at high $p_{T}$ in the recombination scenario, and (d) the effect is expected to be larger for $K^{* 0}$ compared to $\phi$ due to their constituent quark composition. The initial large magnetic field might also affect the $\rho_{00}$ values [15]. This leads to $\rho_{00}>1 / 3$ for neutral and $\rho_{00}<1 / 3$ for charged vector mesons. Hence magnetic field and angular momentum could have opposite effects on electrically neutral $K^{* 0}, \phi$. All of these features are probed for $K^{* 0}$ and $\phi$ mesons in $\mathrm{Pb}-\mathrm{Pb}$ collisions presented in this Letter. As a cross check, a control measurement is carried out using $p p$ collisions, which do not possess large initial angular momentum, and the same analysis is done in $\mathrm{Pb}-\mathrm{Pb}$ collisions for $K_{S}^{0}$ meson, which has zero spin. In addition, the measurements are carried out by randomizing the directions of the event (RNDEP) and production planes.

The analyses are carried out using 43 million minimum bias $p p$ collisions at $\sqrt{s}=13 \mathrm{TeV}$, taken in 2015 and 14 million minimum bias $\mathrm{Pb}-\mathrm{Pb}$ collisions at $\sqrt{s_{\mathrm{NN}}}=2.76 \mathrm{TeV}$, collected in 2010. The minimum bias event selection in $\mathrm{Pb}-\mathrm{Pb}$ collisions require at least one hit in any of V0A, V0C, and silicon pixel detectors while in $p p$ collisions at least one hit in both V0A and V0C is required. The events are further required to have a primary vertex position within $\pm 10 \mathrm{~cm}$ of the detector center along the beam axis. The events were classified by collision centrality classes based on the amplitude measured in the V0 counters [20]. The measurements are performed at midrapidity $(|y|<0.5)$ as a function of $p_{T}$ and are reported for $p p$ collisions as well as for different centrality classes in $\mathrm{Pb}-\mathrm{Pb}$ collisions. The $K_{S}^{0}$ analysis is performed only for $\mathrm{Pb}-$ $\mathrm{Pb}$ collisions in the $20-40 \%$ centrality class which corresponds to the top $20-40 \%$ of V0 amplitude distribution. The details of the ALICE detector, trigger conditions, centrality selection, and second order event plane estimation using the V0 detectors at forward rapidity, can be found in [20-23]. The $K^{* 0}$ and $\phi$ candidates are reconstructed via their decays into charged $K \pi$ and $K K$ pairs, respectively, while the $K_{S}^{0}$ is reconstructed via its decay into two charged pions. The time projection chamber (TPC) [24] and time-of-flight (TOF) detector [25] are used to identify the decay products of these mesons via specific ionization energy loss and time-of-flight measurements, respectively. The $K^{* 0}$ and $\phi$ yields are determined via the invariant mass technique [26-28]. The background coming from combinatorial pairs and misidentified particles is removed by constructing the invariant mass distribution from so-called mixed events for the $K^{* 0}$ and $\phi[26,27]$. The combinatorial background for the $K_{S}^{0}$ candidates is significantly reduced by selecting the distinctive $V$-shaped decay topology [28].

The invariant mass distributions are fitted with a BreitWigner and Voigtian (convolution of Breit-Wigner and Gaussian distributions) function for the $K^{* 0}$ and $\phi$ signals, respectively, along with a second-order polynomial that describes the residual background [26,27]. Extracted yields are then corrected for the reconstruction efficiency and acceptance in each $\cos \theta^{*}$ and $p_{T}$ bin [26,27]. The reconstruction efficiency is determined from Monte Carlo simulations of the ALICE detector response based on GEANT3 simulation $[26,27]$. The signal extraction procedures for the vector mesons and $K_{S}^{0}$ are identical to those used in earlier publications reporting the $p_{T}$ distribution of the mesons [26-28]. The mass peak positions and widths of the resonances across all the $\cos \theta^{*}$ bins for various $p_{T}$ intervals in $p p$ collisions and in different centrality classes of $\mathrm{Pb}-\mathrm{Pb}$ collisions are consistent with those obtained from earlier analyses [26-28] and no significant dependence on $\cos \theta^{*}$ is seen. The resulting efficiency and acceptance corrected $d N / d \cos \theta^{*}$ distributions for selected $p_{T}$ intervals in minimum bias $p p$ collisions and in $10-50 \%$ central $\mathrm{Pb}-\mathrm{Pb}$ collisions are shown in Fig. 1. These distributions are fitted with the functional form given in Eq. (1) to determine $\rho_{00}$ for each $p_{T}$ bin in $p p$ and $\mathrm{Pb}-\mathrm{Pb}$ collisions. For the EP results, the resolution values $R$ are $0.71,0.53$, $0.72,0.66$, and 0.40 for $10-50 \%, 0-10 \%, 10-30 \%$,

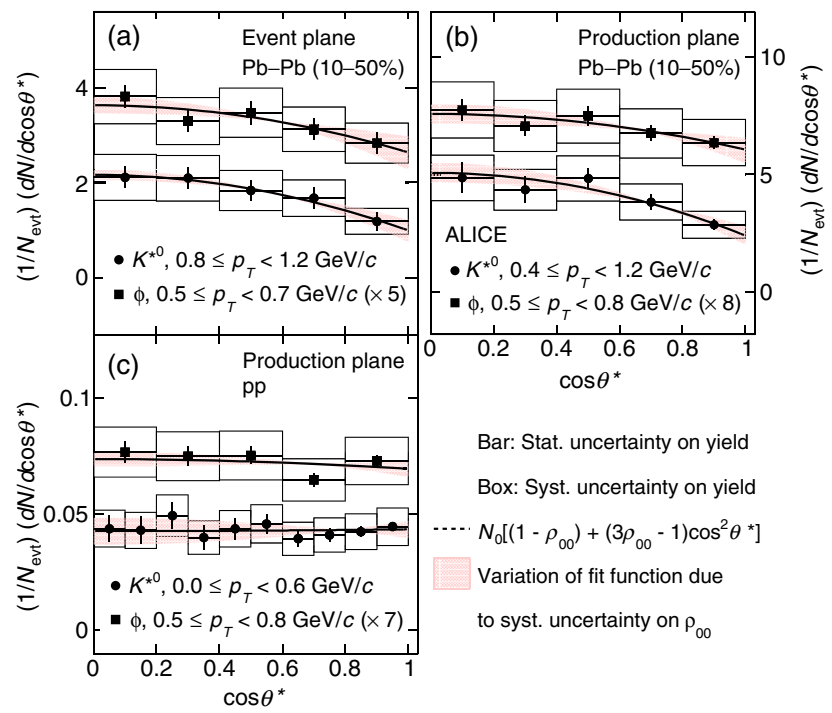

FIG. 1. Angular distribution of the decay daughter in the rest frame of the meson with respect to the quantization axis at $|y|<$ 0.5 for $p p$ collisions at $\sqrt{s}=13 \mathrm{TeV}$ and $\mathrm{Pb}-\mathrm{Pb}$ collisions at $\sqrt{s_{\mathrm{NN}}}=2.76 \mathrm{TeV}$. Panels (a) and (b) show results for $K^{* 0}$ and $\phi$ with respect to EP and PP. Panel (c) is the results for vector mesons in $p p$ collisions with respect to PP. 
$30-50 \%$, and $50-80 \%$ collision centralities, respectively [29].

There are three main sources of systematic uncertainties in the measurements of the angular distribution of vector meson decays. (i) Meson yield extraction: this contribution is estimated by varying the fit ranges for the yield extraction, the normalization range for the signal + background and background invariant mass distributions, the procedure to integrate the signal function to get the yields, and by leaving the width of the resonance peak free or keeping it fixed to the PDG value as discussed in Refs. [26,27]. The uncertainties for $\rho_{00}$ is at a level of $12(8) \%$ at the lowest $p_{T}$ and decrease with $p_{T}$ to $4(3) \%$ at the highest $p_{T}$ studied for the $K^{* 0}(\phi)$. (ii) Track selection: this contribution includes variations of the selection on the distance of closest approach to the collision vertex, the number of crossed pad rows in the TPC [24], the ratio of found clusters to the expected clusters, and the quality of the track fit. The systematic uncertainties for $\rho_{00}$ are $14(6) \%$ at the lowest $p_{T}$ and about $11(5) \%$ at the highest $p_{T}$ for $K^{* 0}(\phi)$. (iii) Particle identification: this is evaluated by varying the particle identification criteria related to the TPC and TOF detectors. The corresponding uncertainty is 5(3)\% at the lowest $p_{T}$ and about 4(4.5)\% at the highest $p_{T}$ studied for $K^{* 0}(\phi)$. Systematic uncertainties due to different variations are considered as uncorrelated and the total systematic uncertainty on $\rho_{00}$ is obtained by adding all the contributions in quadrature. Several consistency checks are carried out and details can be found in the Supplemental Material [17]. The final measurement is reported for the average yield of particles $\left(K^{* 0}\right)$ and antiparticles $\left(\bar{K}^{* 0}\right)$ as results for $K^{* 0}$ and $\bar{K}^{* 0}$ were consistent.

Figure 2 shows the measured $\rho_{00}$ as a function of $p_{T}$ for $K^{* 0}$ and $\phi$ mesons in $p p$ collisions and $\mathrm{Pb}-\mathrm{Pb}$ collisions, along with the measurements for $K_{S}^{0}$ in $\mathrm{Pb}-\mathrm{Pb}$ collisions. In mid-central (10-50\%) $\mathrm{Pb}-\mathrm{Pb}$ collisions, $\rho_{00}$ is below $1 / 3$ at the lowest measured $p_{T}$ and increases to $1 / 3$ within uncertainties for $p_{T}>2 \mathrm{GeV} / c$. At low $p_{T}$, the central value of $\rho_{00}$ is smaller for $K^{* 0}$ than for $\phi$, although the results are compatible within uncertainties. In $p p$ collisions, $\rho_{00}$ is independent of $p_{T}$ and equal to $1 / 3$ within uncertainties. For the spin zero hadron $K_{S}^{0}, \rho_{00}$ is consistent with $1 / 3$ within uncertainties in $\mathrm{Pb}-\mathrm{Pb}$ collisions. The results with random event plane directions are also compatible with no spin alignment for the studied $p_{T}$ range, except for the smallest $p_{T}$ bin, where $\rho_{00}$ less than $1 / 3$ but still larger than for EP and PP measurements. The results for the random production plane (the momentum vector direction of each vector meson is randomized) are similar to RNDEP measurements. These results indicate that a spin alignment is present at lower $p_{T}$, which is a qualitatively consistent with predictions [13].

Figure 3 shows $\rho_{00}$ for $K^{* 0}$ and $\phi$ mesons as a function of average number of participating nucleons $\left(\left\langle N_{\text {part }}\right\rangle\right)[20,22]$ for $\mathrm{Pb}-\mathrm{Pb}$ collisions at $\sqrt{s_{\mathrm{NN}}}=2.76 \mathrm{TeV}$. Large $\left\langle N_{\text {part }}\right\rangle$

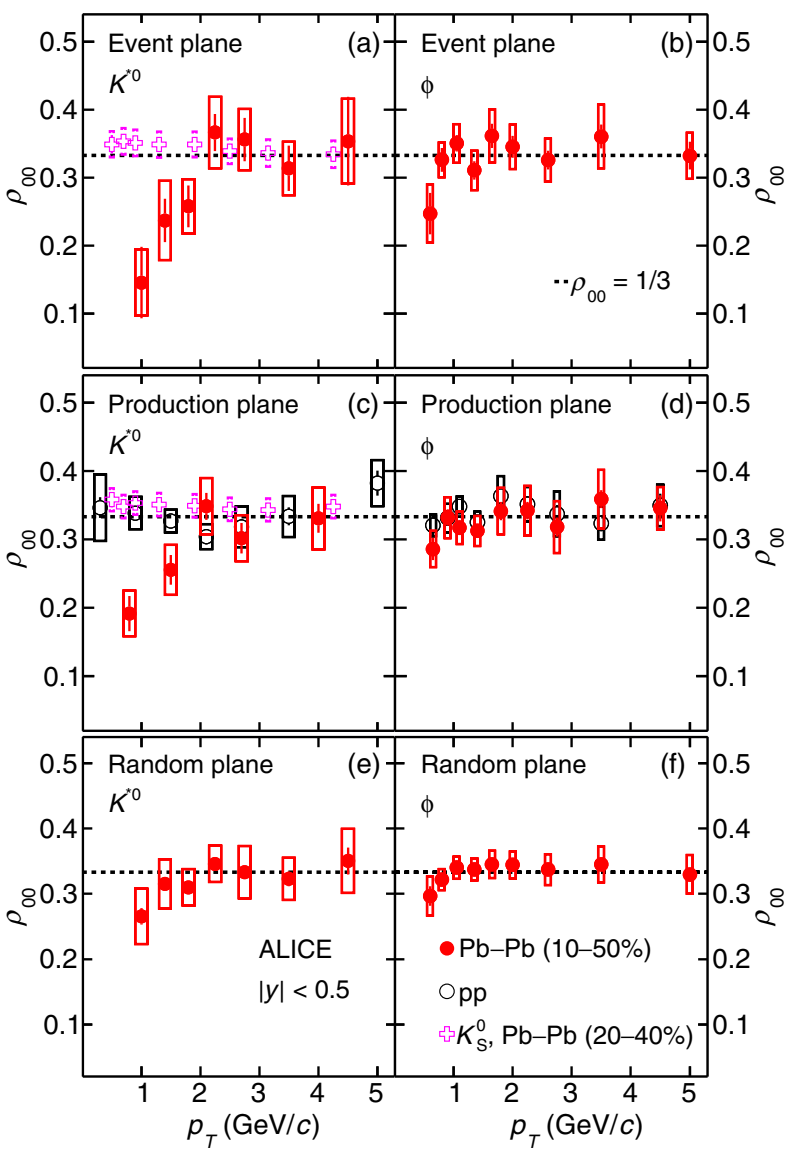

FIG. 2. Transverse momentum dependence of $\rho_{00}$ for $K^{* 0}, \phi$, and $K_{S}^{0}$ mesons at $|y|<0.5$ in $\mathrm{Pb}-\mathrm{Pb}$ collisions at $\sqrt{s_{\mathrm{NN}}}=$ $2.76 \mathrm{TeV}$ and minimum bias $p p$ collisions at $\sqrt{s}=13 \mathrm{TeV}$. Results are shown for spin alignment with respect to the event plane [panels (a),(b)], production plane [(c),(d)], and random event plane [(e),(f)] for $K^{* 0}$ (left column) and $\phi$ (right column). The statistical and systematic uncertainties are shown as bars and boxes, respectively.

correspond to central collisions and small $\left\langle N_{\text {part }}\right\rangle$ correspond to peripheral collisions (see Table I of the Supplemental Material [17]). In the lowest $p_{T}$ range, $\rho_{00}$ shows maximum deviation from $1 / 3$ for intermediate centrality and approaches $1 / 3$ for both central and peripheral collisions. This centrality dependence is qualitatively consistent with the dependence of the initial angular momentum on impact parameter in heavy-ion collisions [4]. At higher $p_{T}, \rho_{00}$ is consistent with $1 / 3$ for all centrality classes. For the low- $p_{T}$ measurements in 10$30 \%$ (20-40\% for $\phi$ meson with respect to PP) mid-central $\mathrm{Pb}-\mathrm{Pb}$ collisions, the maximum deviations of $\rho_{00}$ from $1 / 3$ with respect to the PP (EP) are 3.2 (2.6) $\sigma$ and 2.1 (1.9) $\sigma$ for $K^{* 0}$ and $\phi$ mesons, respectively. The errors $(\sigma)$ are calculated by adding statistical and systematic uncertainties in quadrature.

The relation between the $\rho_{00}$ values with respect to different quantization axes can be expressed using Eq. (2) 


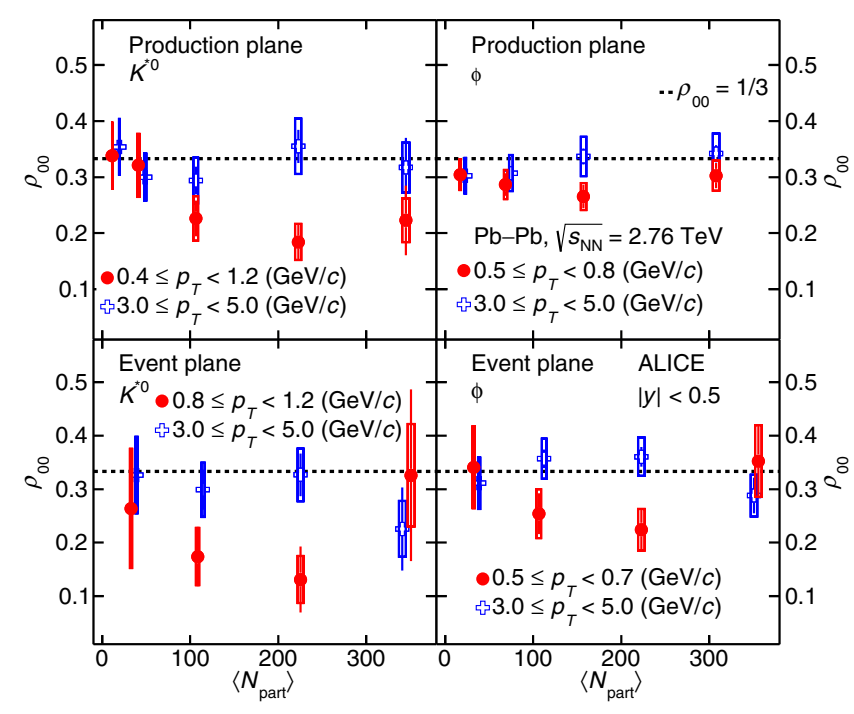

FIG. 3. Measurements of $\rho_{00}$ as a function of $\left\langle N_{\text {part }}\right\rangle$ for $K^{* 0}$ and $\phi$ mesons at low and high $p_{T}$ in $\mathrm{Pb}-\mathrm{Pb}$ collisions. The statistical and systematic uncertainties are shown as bars and boxes, respectively. Some data points are shifted horizontally for better visibility.

and calculating the corresponding factor $R$. This gives $\Delta \rho_{00}(R N D E P)=\Delta \rho_{00}(E P) \times \frac{1}{4}(R=0$ for random plane $)$ and $\Delta \rho_{00}(\mathrm{PP})=\Delta \rho_{00}(\mathrm{EP}) \times\left(1+3 v_{2}\right) / 4\left(R=v_{2}\right.$ for production plane, where $v_{2}$ is the second Fourier coefficient of the azimuthal distribution of produced particles relative to the event plane angle). Here $\Delta \rho_{00}=\rho_{00}-1 / 3$. This is further confirmed using a toy model simulation with the PYTHIA 8.2 event generator [30] by incorporating $v_{2}$ and spin alignment (see the Supplemental Material [17] for further details).

In the past, spin alignment measurements in $e^{+} e^{-}$ [31-33], hadron-proton [34] and nucleon-nucleus collisions [35] were carried out to understand the role of spin in the dynamics of particle production, finding $\rho_{00}>1 / 3$ and off-diagonal elements close to zero with respect to the PP. For $p p$ collisions at $\sqrt{s}=13 \mathrm{TeV}$, we find $\rho_{00} \sim 1 / 3$ within the studied $p_{T}$ range (see Fig. 2). New preliminary results from RHIC have found deviations of $\rho_{00}$ from $1 / 3$ indicating spin alignment for vector mesons at lower $\sqrt{s_{\mathrm{NN}}}$ [36,37]. The $\rho_{00}$ for $\phi$ mesons in mid-central $\mathrm{Pb}-\mathrm{Pb}$ collisions at $\sqrt{s_{\mathrm{NN}}}=2.76 \mathrm{TeV}$ is less than $1 / 3$ while the preliminary finding for mid-central $\mathrm{Au}-\mathrm{Au}$ collisions at $\sqrt{s_{\mathrm{NN}}}=200 \mathrm{GeV}$ is $\rho_{00}$ greater than $1 / 3$. The $\rho_{00}>1 / 3$ for $\phi$ mesons has been interpreted as evidence for a coherent $\phi$ meson field [38]. Similar conclusions cannot be easily applied to $K^{* 0}$ as it consists of valence quarks of unequal mass $(s$ and $\bar{d})$, which makes it impossible to separate the effects of vorticity and due to electromangetic and mesonic fields. Significant polarization of $\Lambda$ baryons ( $\operatorname{spin}=1 / 2)$ was reported at low RHIC energies. The polarization is found to decrease with increasing $\sqrt{s_{\mathrm{NN}}}$
$[39,40]$. At the LHC, the global polarization for $\Lambda$ baryon is compatible with zero within uncertainties $\left[P_{\Lambda}(\%)=\right.$ $0.01 \pm 0.06 \pm 0.03$ ] [41]. The spin alignment for vector mesons in heavy ion collisions could have contributions from angular momentum [12,13], electromagnetic fields [15] and mesonic fields [38]. While no quantitative theoretical calculation for vector meson polarization at LHC energies exists, the expected order of magnitude can be estimated and the measurements for vector mesons and hyperons can be related in a model dependent way. Considering only the angular momentum contribution and recombination as the process of hadronization [13], the $\rho_{00}$ of vector mesons are related to quark polarization as $\rho_{00}=\left(1-P_{q} P_{\bar{q}}\right) /\left(3+P_{q} P_{\bar{q}}\right)$ where $P_{q}$ and $P_{\bar{q}}$ are quark and antiquark polarization, respectively. Assuming $P_{u}=$ $P_{\bar{u}}=P_{d}=P_{\bar{d}}$ and $P_{s}=P_{\bar{s}}$, the measured $p_{T}$ integrated $\rho_{00}$ values for $K^{* 0}$ and $\phi$ mesons in $10-50 \% \mathrm{~Pb}-\mathrm{Pb}$ collisions could translate to light quark polarization of $\sim 0.8$ and strange quark polarization of $\sim 0.2$. Using a thermal and nonrelativistic approach as discussed in [42], vorticity $(\omega)$ and temperature $(T)$ are related to $\rho_{00}$ and vector meson polarization $\left(P_{V}\right)$ as $\rho_{00} \simeq \frac{1}{3}\left\{1-\left[(\omega / T)^{2} / 3\right]\right\}$ and $P_{V} \simeq(2 \omega / 3 T)$, respectively. Also in this approach, the measured $\rho_{00}$ for $K^{* 0}$ would correspond to $K^{* 0}$ polarization of $\sim 0.6$ and the $\rho_{00}$ for $\phi$ mesons would give $\phi$ meson polarization of $\sim 0.3$.

In the recombination model, $\Lambda$ polarization depends linearly on quark polarization whereas vector meson polarization depends quadratically on it. One would therefore expect the polarization for $K^{* 0}$ to be of the same order or smaller than the one measured for the $\Lambda$ at LHC [41], i.e., vanishing small $[O(0.01 \%)]$ rather than order 1 . The large effect observed for the $\rho_{00}$ in mid-central $\mathrm{Pb}-\mathrm{Pb}$ collisions at low $p_{T}$ is therefore puzzling. This result should stimulate further theoretical work in order to study which effects could make such a huge difference between $\Lambda$ and $K^{* 0}$ polarization. Possible reasons may include the transfer of the quark polarization to the hadrons (baryon vs meson), details of the hadronization mechanism (recombination vs fragmentation), rescattering, regeneration, and possibly the lifetime and mass of the relevant hadron. Moreover, the vector mesons are predominantly directly produced whereas the hyperons have large contributions from resonance decays.

In conclusion, for the first time, evidence has been found for a significant spin alignment of vector mesons in heavyion collisions. The effect is strongest at low $p_{T}$ with respect to a vector perpendicular to the reaction plane and for midcentral (10-50\%) collisions. These observations are qualitatively consistent with expectations from the effect of large initial angular momentum in noncentral heavy-ion collisions, which leads to quark polarization via spin-orbit coupling, subsequently transferred to hadronic degrees of freedom by hadronization via recombination. However, the measured spin alignment is surprisingly large compared to 
the polarization measured for $\Lambda$ hyperons where, in addition, a strong decrease in polarization with $\sqrt{s_{\mathrm{NN}}}$ is observed. In future measurements, the difference in the polarization of $K^{* \pm}$ and $K^{* o}$, due to their difference in magnetic moment, would be directly sensitive to the effect of the large initial magnetic field produced in heavy-ion collisions.

The ALICE Collaboration would like to thank all its engineers and technicians for their invaluable contributions to the construction of the experiment and the CERN accelerator teams for the outstanding performance of the LHC complex. The ALICE Collaboration gratefully acknowledges the resources and support provided by all Grid centres and the Worldwide LHC Computing Grid (WLCG) collaboration. The ALICE Collaboration acknowledges the following funding agencies for their support in building and running the ALICE detector: A. I. Alikhanyan National Science Laboratory (Yerevan Physics Institute) Foundation (ANSL), State Committee of Science and World Federation of Scientists (WFS), Armenia; Austrian Academy of Sciences, Austrian Science Fund (FWF): [M 2467-N36] and Nationalstiftung für Forschung, Technologie und Entwicklung, Austria; Ministry of Communications and High Technologies, National Nuclear Research Center, Azerbaijan; Conselho Nacional de Desenvolvimento Científico e Tecnológico (CNPq), Financiadora de Estudos e Projetos (Finep), Fundação de Amparo à Pesquisa do Estado de São Paulo (FAPESP) and Universidade Federal do Rio Grande do Sul (UFRGS), Brazil; Ministry of Education of China (MOEC), Ministry of Science \& Technology of China (MSTC) and National Natural Science Foundation of China (NSFC), China; Ministry of Science and Education and Croatian Science Foundation, Croatia; Centro de Aplicaciones Tecnológicas y Desarrollo Nuclear (CEADEN), Cubaenergía, Cuba; Ministry of Education, Youth and Sports of the Czech Republic, Czech Republic; The Danish Council for Independent Research | Natural Sciences, the VILLUM FONDEN and Danish National Research Foundation (DNRF), Denmark; Helsinki Institute of Physics (HIP), Finland; Commissariat à l'Energie Atomique (CEA), Institut National de Physique Nucléaire et de Physique des Particules (IN2P3) and Centre National de la Recherche Scientifique (CNRS) and Région des Pays de la Loire, France; Bundesministerium für Bildung und Forschung (BMBF) and GSI Helmholtzzentrum für Schwerionenforschung GmbH, Germany; General Secretariat for Research and Technology, Ministry of Education, Research and Religions, Greece; National Research, Development and Innovation Office, Hungary; Department of Atomic Energy Government of India (DAE), Department of Science and Technology, Government of India (DST), University Grants Commission, Government of India (UGC) and Council of Scientific and Industrial Research (CSIR), India;
Indonesian Institute of Science, Indonesia; Centro Fermi - Museo Storico della Fisica e Centro Studi e Ricerche Enrico Fermi and Istituto Nazionale di Fisica Nucleare (INFN), Italy; Institute for Innovative Science and Technology, Nagasaki Institute of Applied Science (IIST), Japanese Ministry of Education, Culture, Sports, Science and Technology (MEXT) and Japan Society for the Promotion of Science (JSPS) KAKENHI, Japan; Consejo Nacional de Ciencia (CONACYT) y Tecnología, through Fondo de Cooperación Internacional en Ciencia y Tecnología (FONCICYT) and Dirección General de Asuntos del Personal Academico (DGAPA), Mexico; Nederlandse Organisatie voor Wetenschappelijk Onderzoek (NWO), Netherlands; The Research Council of Norway, Norway; Commission on Science and Technology for Sustainable Development in the South (COMSATS), Pakistan; Pontificia Universidad Católica del Perú, Peru; Ministry of Science and Higher Education and National Science Centre, Poland; Korea Institute of Science and Technology Information and National Research Foundation of Korea (NRF), Republic of Korea; Ministry of Education and Scientific Research, Institute of Atomic Physics and Ministry of Research and Innovation and Institute of Atomic Physics, Romania; Joint Institute for Nuclear Research (JINR), Ministry of Education and Science of the Russian Federation, National Research Centre Kurchatov Institute, Russian Science Foundation and Russian Foundation for Basic Research, Russia; Ministry of Education, Science, Research and Sport of the Slovak Republic, Slovakia; National Research Foundation of South Africa, South Africa; Swedish Research Council (VR) and Knut \& Alice Wallenberg Foundation (KAW), Sweden; European Organization for Nuclear Research, Switzerland; Suranaree University of Technology (SUT), National Science and Technology Development Agency (NSDTA) and Office of the Higher Education Commission under NRU project of Thailand, Thailand; Turkish Atomic Energy Agency (TAEK), Turkey; National Academy of Sciences of Ukraine, Ukraine; Science and Technology Facilities Council (STFC), United Kingdom; National Science Foundation of the United States of America (NSF) and United States Department of Energy, Office of Nuclear Physics (DOE NP), United States of America.

[1] J. Adams et al. (STAR Collaboration), Experimental and theoretical challenges in the search for the quark gluon plasma: The STAR Collaboration's critical assessment of the evidence from RHIC collisions, Nucl. Phys. A757, 102 (2005).

[2] M. Gyulassy and L. McLerran, New forms of QCD matter discovered at RHIC, Nucl. Phys. A750, 30 (2005).

[3] A. Andronic, P. Braun-Munzinger, K. Redlich, and J. Stachel, Decoding the phase structure of QCD via particle 
production at high energy, Nature (London) 561, 321 (2018).

[4] F. Becattini, F. Piccinini, and J. Rizzo, Angular momentum conservation in heavy ion collisions at very high energy, Phys. Rev. C 77, 024906 (2008).

[5] D. E. Kharzeev, L. D. McLerran, and H. J. Warringa, The effects of topological charge change in heavy ion collisions: 'Event by event $\mathrm{P}$ and $C P$ violation', Nucl. Phys. A803, 227 (2008).

[6] R. J. Fries, G. Chen, and S. Somanathan, Initial Angular Momentum and Flow in High Energy Nuclear Collisions, Phys. Rev. C 97, 034903 (2018).

[7] V. Voronyuk, V. D. Toneev, W. Cassing, E. L. Bratkovskaya, V.P. Konchakovski, and S. A. Voloshin, Electromagnetic field evolution in relativistic heavy-ion collisions, Phys. Rev. C 83, 054911 (2011).

[8] J. D. Jackson, Classical Electrodynamics, 3rd ed. (Wiley, New York, 1982), Secs. 11-8 and 11-11.

[9] V. B. Berestetskii, E. M. Lifshitz, and L. P. Pitaevskii, Quantum Electrodynamics, Volume 4 of Course of Theoretical Physics, 2nd ed. (Butterworth-Heinemann, Oxford, 1982).

[10] M. G. Mayer, On closed shells in nuclei. II, Phys. Rev. 75, 1969 (1949).

[11] S. A. Voloshin, Polarized secondary particles in unpolarized high energy hadron-hadron collisions?, arXiv:nucl-th/ 0410089 .

[12] Z.-T. Liang and X.-N. Wang, Globally Polarized QuarkGluon Plasma in Non-Central A + A Collisions, Phys. Rev. Lett. 94, 102301 (2005); Erratum, Phys. Rev. Lett. 96, 039901 (2006).

[13] Z.-T. Liang and X.-N. Wang, Spin alignment of vector mesons in non-central A + A collisions, Phys. Lett. B 629, 20 (2005).

[14] Z.-T. Liang, Global polarization of QGP in non-central heavy ion collisions at high energies, J. Phys. G 34, S323 (2007).

[15] Y.-G. Yang, R.-H. Fang, Q. Wang, and X.-N. Wang, Quark coalescence model for polarized vector mesons and baryons, Phys. Rev. C 97, 034917 (2018).

[16] K. Schilling, P. Seyboth, and G. E. Wolf, On the analysis of vector meson production by polarized photons, Nucl. Phys. B15, 397 (1970); Erratum, Nucl. Phys. B18, 332 (1970).

[17] See the Supplemental Material at http://link.aps.org/ supplemental/10.1103/PhysRevLett.125.012301 for complete angular distribution, consistency checks, analytical relation between EP and PP, toy model simulation to show the relation between EP, PP and RNDEP, and centrality classes.

[18] A. M. Poskanzer and S. A. Voloshin, Methods for analyzing anisotropic flow in relativistic nuclear collisions, Phys. Rev. C 58, 1671 (1998).

[19] A. H. Tang, B. Tu, and C. S. Zhou, Practical considerations for measuring global spin alignment of vector mesons in relativistic heavy ion collisions, Phys. Rev. C 98, 044907 (2018).

[20] B. Abelev et al. (ALICE Collaboration), Centrality determination of $\mathrm{Pb}-\mathrm{Pb}$ collisions at $\sqrt{s_{N N}}=2.76 \mathrm{TeV}$ with ALICE, Phys. Rev. C 88, 044909 (2013).
[21] K. Aamodt et al., The ALICE experiment at the CERN LHC, J. Instrum. 3, S08002 (2008).

[22] K. Aamodt et al. (ALICE Collaboration), Centrality Dependence of the Charged-Particle Multiplicity Density at Mid-Rapidity in $\mathrm{Pb}-\mathrm{Pb}$ Collisions at $\sqrt{s_{N N}}=2.76 \mathrm{TeV}$, Phys. Rev. Lett. 106, 032301 (2011).

[23] B. B. Abelev et al. (ALICE Collaboration), Elliptic flow of identified hadrons in $\mathrm{Pb}-\mathrm{Pb}$ collisions at $\sqrt{s_{\mathrm{NN}}}=$ 2.76 TeV, J. High Energy Phys. 06 (2015) 190.

[24] J. Alme et al., The ALICE TPC, a large 3-dimensional tracking device with fast readout for ultra-high multiplicity events, Nucl. Instrum. Methods Phys. Res., Sect. A 622, 316 (2010).

[25] G. Dellacasa et al. (ALICE Collaboration), ALICE technical design report of the time-of-flight system (TOF), Report No. CERN-LHCC-2000-012 (2000).

[26] J. Adam et al. (ALICE Collaboration), $K^{*}(892)^{0}$ and $\phi(1020)$ meson production at high transverse momentum in $p p$ and $\mathrm{Pb}-\mathrm{Pb}$ collisions at $\sqrt{s_{\mathrm{NN}}}=2.76 \mathrm{TeV}$, Phys. Rev. C 95, 064606 (2017).

[27] B. B. Abelev et al. (ALICE Collaboration), $K^{*}(892)^{0}$ and $\phi(1020)$ production in $\mathrm{Pb}-\mathrm{Pb}$ collisions at $\sqrt{s_{\mathrm{NN}}}=$ 2.76 TeV, Phys. Rev. C 91, 024609 (2015).

[28] B. B. Abelev et al. (ALICE Collaboration), $K_{\mathrm{S}}^{0}$ and $\Lambda$ Production in $\mathrm{Pb}-\mathrm{Pb}$ Collisions at $\sqrt{s_{N N}}=2.76 \mathrm{TeV}$, Phys. Rev. Lett. 111, 222301 (2013).

[29] B. B. Abelev et al. (ALICE Collaboration), Performance of the ALICE Experiment at the CERN LHC, Int. J. Mod. Phys. A 29, 1430044 (2014).

[30] T. Sjöstrand, S. Ask, J. R. Christiansen, R. Corke, N. Desai, P. Ilten, S. Mrenna, S. Prestel, C. O. Rasmussen, and P. Z. Skands, An Introduction to PYTHIA 8.2, Comput. Phys. Commun. 191, 159 (2015).

[31] K. Ackerstaff et al. (OPAL Collaboration), Spin alignment of leading $\mathrm{K}^{* 0}(892)$ mesons in hadronic $\mathrm{Z}^{0}$ decays, Phys. Lett. B 412, 210 (1997).

[32] K. Ackerstaff et al. (OPAL Collaboration), Study of $\phi(1020), \mathrm{D}^{* \pm}$ and $\mathrm{B}^{*}$ spin alignment in hadronic $\mathrm{Z}^{0}$ decays, Z. Phys. C 74, 437 (1997).

[33] P. Abreu et al. (DELPHI Collaboration), Measurement of the spin density matrix for the $\rho^{0}, K^{* 0}(892)$ and $\phi$ produced in $Z^{0}$ decays, Phys. Lett. B 406, 271 (1997).

[34] M. Barth et al. (Brussels-Genoa-Mons-NijmegenSerpukhov-CERN Collaborations), Inclusive resonance production in $K^{+}$p interactions at $70-\mathrm{GeV} / c$, Nucl. Phys. B223, 296 (1983); Erratum, Nucl. Phys. B232, 547 (1984).

[35] A. N. Aleev et al. (EXCHARM Collaboration), Spin alignment of $K^{* \pm}(892)$ mesons produced in neutron carbon interactions, Phys. Lett. B 485, 334 (2000).

[36] C. Zhou, $\phi$ meson and $K^{* 0}$ global spin alignment at STAR, Nucl. Phys. A982, 559 (2019).

[37] B. I. Abelev et al. (STAR Collaboration), Spin alignment measurements of the $K^{* 0}(892)$ and $\phi(1020)$ vector mesons in heavy ion collisions at $\sqrt{s_{\mathrm{NN}}}=200 \mathrm{GeV}$, Phys. Rev. C 77, 061902 (2008).

[38] X.-L. Sheng, L. Oliva, and Q. Wang, What can we learn from global spin alignment of $\phi$ meson in heavy-ion collisions?, Phys. Rev. D 101, 096005 (2020). 
[39] L. Adamczyk et al. (STAR Collaboration), Global $\Lambda$ hyperon polarization in nuclear collisions: Evidence for the most vortical fluid, Nature (London) 548, 62 (2017).

[40] J. Adam et al. (STAR Collaboration), Global polarization of $\Lambda$ hyperons in $\mathrm{Au}+\mathrm{Au}$ collisions at $\sqrt{s_{N N}}=200 \mathrm{GeV}$, Phys. Rev. C 98, 014910 (2018).
[41] S. Acharya et al. (ALICE Collaboration), Global polarization of $\Lambda \bar{\Lambda}$ hyperons in $\mathrm{Pb}-\mathrm{Pb}$ collisions at $\sqrt{s_{N N}}=2.76$ and 5.02 TeV, Phys. Rev. C 101, 044611 (2020).

[42] F. Becattini, I. Karpenko, M. Lisa, I. Upsal, and S. Voloshin, Global hyperon polarization at local thermodynamic equilibrium with vorticity, magnetic field and feed-down, Phys. Rev. C 95, 054902 (2017).

S. Acharya, ${ }^{141}$ D. Adamová, ${ }^{94}$ A. Adler, ${ }^{74}$ J. Adolfsson, ${ }^{80}$ M. M. Aggarwal, ${ }^{99}$ G. Aglieri Rinella, ${ }^{33}$ M. Agnello, ${ }^{30}$ N. Agrawal, ${ }^{10,53}$ Z. Ahammed, ${ }^{141}$ S. Ahmad, ${ }^{16}$ S. U. Ahn, ${ }^{76}$ A. Akindinov, ${ }^{91}$ M. Al-Turany, ${ }^{106}$ S. N. Alam, ${ }^{141}$ D. S. D. Albuquerque, ${ }^{122}$ D. Aleksandrov, ${ }^{87}$ B. Alessandro, ${ }^{58}$ H. M. Alfanda, ${ }^{6}$ R. Alfaro Molina, ${ }^{71}$ B. Ali, ${ }^{16}$ Y. Ali, ${ }^{14}$ A. Alici, ${ }^{10,26,53}$ A. Alkin, ${ }^{2}$ J. Alme,${ }^{21}$ T. Alt,${ }^{68}$ L. Altenkamper, ${ }^{21}$ I. Altsybeev, ${ }^{112}$ M. N. Anaam, ${ }^{6}$ C. Andrei,${ }^{47}$ D. Andreou, ${ }^{33}$ H. A. Andrews, ${ }^{110}$ A. Andronic, ${ }^{144}$ M. Angeletti, ${ }^{33}$ V. Anguelov, ${ }^{103}$ C. Anson, ${ }^{15}$ T. Antičić, ${ }^{107}$ F. Antinori, ${ }^{56}$ P. Antonioli,${ }^{53}$ R. Anwar, ${ }^{125}$ N. Apadula ${ }^{79}$ L. Aphecetche,${ }^{114}$ H. Appelshäuser, ${ }^{68}$ S. Arcelli, ${ }^{26}$ R. Arnaldi,${ }^{58}$ M. Arratia,${ }^{79}$ I. C. Arsene,${ }^{20}$ M. Arslandok, ${ }^{103}$ A. Augustinus, ${ }^{33}$ R. Averbeck, ${ }^{106}$ S. Aziz,${ }^{61}$ M. D. Azmi, ${ }^{16}$ A. Badalà, ${ }^{55}$ Y. W. Baek, ${ }^{40}$ S. Bagnasco, ${ }^{58}$

X. Bai ${ }^{106}$ R. Bailhache, ${ }^{68}$ R. Bala ${ }^{100}$ A. Baldisseri, ${ }^{137}$ M. Ball, ${ }^{42}$ S. Balouza, ${ }^{104}$ R. Barbera,${ }^{27}$ L. Barioglio, ${ }^{25}$ G. G. Barnaföldi, ${ }^{145}$ L. S. Barnby, ${ }^{93}$ V. Barret, ${ }^{134}$ P. Bartalini, ${ }^{6}$ K. Barth,${ }^{33}$ E. Bartsch,${ }^{68}$ F. Baruffaldi, ${ }^{28}$ N. Bastid, ${ }^{134}$ S. Basu, ${ }^{143}$ G. Batigne, ${ }^{114}$ B. Batyunya, ${ }^{75}$ D. Bauri, ${ }^{48}$ J. L. Bazo Alba, ${ }^{111}$ I. G. Bearden ${ }^{88}$ C. Bedda ${ }^{63}$ N. K. Behera, ${ }^{60}$ I. Belikov, ${ }^{136}$ A. D. C. Bell Hechavarria ${ }^{144}$ F. Bellini, ${ }^{33}$ R. Bellwied, ${ }^{125}$ V. Belyaev, ${ }^{92}$ G. Bencedi, ${ }^{145}$ S. Beole, ${ }^{25}$ A. Bercuci, ${ }^{47}$ Y. Berdnikov, ${ }^{97}$ D. Berenyi, ${ }^{145}$ R. A. Bertens, ${ }^{130}$ D. Berzano, ${ }^{58}$ M. G. Besoiu ${ }^{67}$ L. Betev,${ }^{33}$ A. Bhasin, ${ }^{100}$ I. R. Bhat, ${ }^{100}$ M. A. Bhat,${ }^{3}$ H. Bhatt, ${ }^{48}$ B. Bhattacharjee, ${ }^{41}$ A. Bianchi, ${ }^{25}$ L. Bianchi, ${ }^{25}$ N. Bianchi, ${ }^{51}$ J. Bielčík, ${ }^{36}$

J. Bielč́́ková, ${ }^{4}$ A. Bilandzic, ${ }^{104,117}$ G. Biro, ${ }^{145}$ R. Biswas, ${ }^{3}$ S. Biswas, ${ }^{3}$ J. T. Blair, ${ }^{119}$ D. Blau, ${ }^{87}$ C. Blume, ${ }^{68}$ G. Boca, ${ }^{139}$ F. Bock,${ }^{33,95}$ A. Bogdanov ${ }^{92}$ S. Boi ${ }^{23}$ L. Boldizsár,${ }^{145}$ A. Bolozdynya,${ }^{92}$ M. Bombara, ${ }^{37}$ G. Bonomi,${ }^{140}$ H. Borel, ${ }^{137}$ A. Borissov, ${ }^{92,144}$ H. Bossi, ${ }^{146}$ E. Botta ${ }^{25}$ L. Bratrud, ${ }^{68}$ P. Braun-Munzinger,${ }^{106}$ M. Bregant, ${ }^{121}$ M. Broz,${ }^{36}$ E. J. Brucken, ${ }^{43}$ E. Bruna ${ }^{58}$ G. E. Bruno, ${ }^{105}$ M. D. Buckland,${ }^{127}$ D. Budnikov, ${ }^{108}$ H. Buesching ${ }^{68}$ S. Bufalino, ${ }^{30}$ O. Bugnon, ${ }^{114}$ P. Buhler, ${ }^{113}$ P. Buncic, ${ }^{33}$ Z. Buthelezi, ${ }^{72,131}$ J. B. Butt, ${ }^{14}$ J. T. Buxton, ${ }^{96}$ S. A. Bysiak, ${ }^{118}$ D. Caffarri, ${ }^{89}$ A. Caliva, ${ }^{106}$ E. Calvo Villar, ${ }^{111}$ R. S. Camacho, ${ }^{44}$ P. Camerini, ${ }^{24}$ A. A. Capon, ${ }^{113}$ F. Carnesecchi, ${ }^{10,26}$ R. Caron, ${ }^{137}$ J. Castillo Castellanos, ${ }^{137}$ A. J. Castro, ${ }^{130}$ E. A. R. Casula, ${ }^{54}$ F. Catalano, ${ }^{30}$ C. Ceballos Sanchez, ${ }^{52}$ P. Chakraborty, ${ }^{48}$ S. Chandra, ${ }^{141}$ W. Chang, ${ }^{6}$ S. Chapeland, ${ }^{33}$ M. Chartier, ${ }^{127}$ S. Chattopadhyay, ${ }^{141}$ S. Chattopadhyay, ${ }^{109}$ A. Chauvin,${ }^{23}$ C. Cheshkov, ${ }^{135}$ B. Cheynis,${ }^{135}$

V. Chibante Barroso, ${ }^{33}$ D. D. Chinellato, ${ }^{122}$ S. Cho, ${ }^{60}$ P. Chochula, ${ }^{33}$ T. Chowdhury, ${ }^{134}$ P. Christakoglou, ${ }^{89}$

C. H. Christensen ${ }^{88}$ P. Christiansen, ${ }^{80}$ T. Chujo, ${ }^{133}$ C. Cicalo,${ }^{54}$ L. Cifarelli, ${ }^{10,26}$ F. Cindolo, ${ }^{53}$ J. Cleymans, ${ }^{124}$ F. Colamaria, ${ }^{52}$ D. Colella, ${ }^{52}$ A. Collu,${ }^{79}$ M. Colocci, ${ }^{26}$ M. Concas, ${ }^{58, b}$ G. Conesa Balbastre, ${ }^{78}$ Z. Conesa del Valle, ${ }^{61}$ G. Contin, ${ }^{24,127}$ J. G. Contreras, ${ }^{36}$ T. M. Cormier, ${ }^{95}$ Y. Corrales Morales, ${ }^{25}$ P. Cortese, ${ }^{31}$ M. R. Cosentino, ${ }^{123}$ F. Costa, ${ }^{33}$ S. Costanza, ${ }^{139}$ P. Crochet, ${ }^{134}$ E. Cuautle, ${ }^{69}$ P. Cui, ${ }^{6}$ L. Cunqueiro, ${ }^{95}$ D. Dabrowski, ${ }^{142}$ T. Dahms, ${ }^{104,117}$ A. Dainese, ${ }^{56}$ F. P. A. Damas, ${ }^{114,137}$ M. C. Danisch,${ }^{103}$ A. Danu, ${ }^{67}$ D. Das, ${ }^{109}$ I. Das, ${ }^{109}$ P. Das,${ }^{85}$ P. Das, ${ }^{3}$ S. Das, ${ }^{3}$ A. Dash, ${ }^{85}$ S. Dash, ${ }^{48}$ S. De ${ }^{85}$ A. De Caro, ${ }^{29}$ G. de Cataldo,${ }^{52}$ J. de Cuveland, ${ }^{38}$ A. De Falco, ${ }^{23}$ D. De Gruttola, ${ }^{10}$ N. De Marco,${ }^{58}$ S. De Pasquale ${ }^{29}$ S. Deb, ${ }^{49}$ B. Debjani, ${ }^{3}$ H. F. Degenhardt, ${ }^{121}$ K. R. Deja, ${ }^{142}$ A. Deloff, ${ }^{84}$ S. Delsanto, ${ }^{25,131}$ D. Devetak, ${ }^{106}$ P. Dhankher, ${ }^{48}$ D. Di Bari, ${ }^{32}$ A. Di Mauro, ${ }^{33}$ R. A. Diaz, ${ }^{8}$ T. Dietel,${ }^{124}$ P. Dillenseger, ${ }^{68}$ Y. Ding, ${ }^{6}$ R. Divià, ${ }^{33}$ D. U. Dixit, ${ }^{19} \varnothing$. Djuvsland,${ }^{21}$ U. Dmitrieva, ${ }^{62}$ A. Dobrin, ${ }^{33,67}$ B. Dönigus, ${ }^{68}$ O. Dordic, ${ }^{20}$ A. K. Dubey, ${ }^{141}$ A. Dubla, ${ }^{16}$ S. Dudi, ${ }^{99}$ M. Dukhishyam, ${ }^{85}$ P. Dupieux, ${ }^{134}$ R. J. Ehlers, ${ }^{146}$ V. N. Eikeland ${ }^{21}$ D. Elia, ${ }^{52}$ H. Engel,${ }^{74}$ E. Epple,${ }^{146}$ B. Erazmus,${ }^{114}$ F. Erhardt, ${ }^{98}$

A. Erokhin, ${ }^{112}$ M. R. Ersdal, ${ }^{21}$ B. Espagnon, ${ }^{61}$ G. Eulisse, ${ }^{33}$ D. Evans, ${ }^{110}$ S. Evdokimov,${ }^{90}$ L. Fabbietti, ${ }^{104,117}$ M. Faggin,${ }^{28}$ J. Faivre, ${ }^{78}$ F. Fan, ${ }^{6}$ A. Fantoni, ${ }^{51}$ M. Fasel,${ }^{95}$ P. Fecchio, ${ }^{30}$ A. Feliciello, ${ }^{58}$ G. Feofilov, ${ }^{112}$ A. Fernández Téllez, ${ }^{44}$ A. Ferrero, ${ }^{137}$ A. Ferretti, ${ }^{25}$ A. Festanti, ${ }^{33}$ V. J. G. Feuillard, ${ }^{103}$ J. Figiel, ${ }^{118}$ S. Filchagin, ${ }^{108}$ D. Finogeev, ${ }^{62}$ F. M. Fionda, ${ }^{21}$ G. Fiorenza, ${ }^{52}$ F. Flor, ${ }^{125}$ S. Foertsch, ${ }^{72}$ P. Foka, ${ }^{106}$ S. Fokin,${ }^{87}$ E. Fragiacomo, ${ }^{59}$ U. Frankenfeld, ${ }^{106}$ U. Fuchs,${ }^{33}$ C. Furget,${ }^{78}$ A. Furs, ${ }^{62}$ M. Fusco Girard, ${ }^{29}$ J. J. Gaardh $\varnothing j e,{ }^{88}$ M. Gagliardi, ${ }^{25}$ A. M. Gago, ${ }^{111}$ A. Gal, ${ }^{136}$ C. D. Galvan, ${ }^{120}$ P. Ganoti, ${ }^{83}$ C. Garabatos, ${ }^{106}$ E. Garcia-Solis, ${ }^{11}$ K. Garg, ${ }^{27}$ C. Gargiulo, ${ }^{33}$ A. Garibli, ${ }^{86}$ K. Garner, ${ }^{144}$ P. Gasik, ${ }^{104,117}$ E. F. Gauger, ${ }^{119}$ M. B. Gay Ducati, ${ }^{70}$ M. Germain, ${ }^{114}$ J. Ghosh, ${ }^{109}$ P. Ghosh,${ }^{141}$ S. K. Ghosh, ${ }^{3}$ P. Gianotti, ${ }^{51}$ P. Giubellino,${ }^{58,106}$ P. Giubilato, ${ }^{28}$ P. Glässel, ${ }^{103}$ D. M. Goméz Coral, ${ }^{71}$ A. Gomez Ramirez, ${ }^{74}$ V. Gonzalez, ${ }^{106}$ P. González-Zamora, ${ }^{44}$ S. Gorbunov, ${ }^{38}$ 
L. Görlich, ${ }^{118}$ S. Gotovac, ${ }^{34}$ V. Grabski, ${ }^{71}$ L. K. Graczykowski, ${ }^{142}$ K. L. Graham, ${ }^{110}$ L. Greiner, ${ }^{79}$ A. Grelli, ${ }^{63}$ C. Grigoras,${ }^{33}$ V. Grigoriev, ${ }^{92}$ A. Grigoryan, ${ }^{1}$ S. Grigoryan, ${ }^{75}$ O. S. Groettvik, ${ }^{21}$ F. Grosa,${ }^{30}$ J. F. Grosse-Oetringhaus, ${ }^{33}$ R. Grosso, ${ }^{106}$ R. Guernane, ${ }^{78}$ M. Guittiere, ${ }^{114}$ K. Gulbrandsen, ${ }^{88}$ T. Gunji ${ }^{132}$ A. Gupta, ${ }^{100}$ R. Gupta, ${ }^{100}$ I. B. Guzman, ${ }^{44}$ R. Haake, ${ }^{146}$ M. K. Habib, ${ }^{106}$ C. Hadjidakis, ${ }^{61}$ H. Hamagaki, ${ }^{81}$ G. Hamar, ${ }^{145}$ M. Hamid, ${ }^{6}$ R. Hannigan, ${ }^{119}$ M. R. Haque, ${ }^{63,85}$ A. Harlenderova, ${ }^{106}$ J. W. Harris, ${ }^{146}$ A. Harton, ${ }^{11}$ J. A. Hasenbichler, ${ }^{33}$ H. Hassan, ${ }^{95}$ D. Hatzifotiadou, ${ }^{10,53}$ P. Hauer, ${ }^{42}$ S. Hayashi, ${ }^{132}$ S. T. Heckel, ${ }^{68,104}$ E. Hellbär, ${ }^{68}$ H. Helstrup, ${ }^{35}$ A. Herghelegiu, ${ }^{47}$ T. Herman, ${ }^{36}$ E. G. Hernandez, ${ }^{44}$ G. Herrera Corral,${ }^{9}$ F. Herrmann, ${ }^{144}$ K. F. Hetland, ${ }^{35}$ T. E. Hilden, ${ }^{43}$ H. Hillemanns, ${ }^{33}$ C. Hills, ${ }^{127}$ B. Hippolyte, ${ }^{136}$ B. Hohlweger, ${ }^{104}$ D. Horak ${ }^{36}$ A. Hornung, ${ }^{68}$ S. Hornung, ${ }^{106}$ R. Hosokawa,${ }^{15,133}$ P. Hristov, ${ }^{33}$ C. Huang, ${ }^{61}$ C. Hughes, ${ }^{130}$ P. Huhn, ${ }^{68}$ T. J. Humanic,${ }^{96}$ H. Hushnud, ${ }^{109}$ L. A. Husova, ${ }^{144}$ N. Hussain, ${ }^{41}$ S. A. Hussain, ${ }^{14}$ D. Hutter, ${ }^{38}$ J. P. Iddon, ${ }^{33,127}$ R. Ilkaev, ${ }^{108}$ M. Inaba, ${ }^{133}$ G. M. Innocenti, ${ }^{33}$ M. Ippolitov, ${ }^{87}$ A. Isakov, ${ }^{94}$ M. S. Islam, ${ }^{109}$ M. Ivanov, ${ }^{106}$ V. Ivanov,${ }^{97}$ V. Izucheev ${ }^{90}$ B. Jacak ${ }^{79}$ N. Jacazio, ${ }^{53}$ P. M. Jacobs ${ }^{79}$ S. Jadlovska, ${ }^{116}$ J. Jadlovsky,${ }^{116}$ S. Jaelani, ${ }^{63}$ C. Jahnke, ${ }^{121}$ M. J. Jakubowska, ${ }^{142}$ M. A. Janik, ${ }^{142}$ T. Janson, ${ }^{74}$ M. Jercic, ${ }^{98}$ O. Jevons,${ }^{110}$ M. Jin, ${ }^{125}$ F. Jonas,${ }^{95,144}$ P. G. Jones,${ }^{110}$ J. Jung ${ }^{68}$

M. Jung, ${ }^{68}$ A. Jusko, ${ }^{110}$ P. Kalinak, ${ }^{64}$ A. Kalweit, ${ }^{33}$ V. Kaplin, ${ }^{92}$ S. Kar, ${ }^{6}$ A. Karasu Uysal, ${ }^{77}$ O. Karavichev, ${ }^{62}$ T. Karavicheva, ${ }^{62}$ P. Karczmarczyk, ${ }^{33}$ E. Karpechev, ${ }^{62}$ A. Kazantsev, ${ }^{87}$ U. Kebschull, ${ }^{74}$ R. Keidel, ${ }^{46}$ M. Keil,${ }^{33}$ B. Ketzer ${ }^{42}$ Z. Khabanova, ${ }^{89}$ A. M. Khan, ${ }^{6}$ S. Khan ${ }^{16}$ S. A. Khan, ${ }^{141}$ A. Khanzadeev,${ }^{97}$ Y. Kharlov, ${ }^{90}$ A. Khatun, ${ }^{16}$ A. Khuntia, ${ }^{118}$ B. Kileng, ${ }^{35}$ B. Kim,${ }^{60}$ B. Kim, ${ }^{133}$ D. Kim, ${ }^{147}$ D. J. Kim, ${ }^{126}$ E. J. Kim,${ }^{73}$ H. Kim, ${ }^{17}{ }^{147}$ J. Kim, ${ }^{147}$ J. S. Kim, ${ }^{40}$ J. Kim, ${ }^{103}$ J. Kim, ${ }^{147}$ J. Kim, ${ }^{73}$ M. Kim, ${ }^{103}$ S. Kim, ${ }^{18}$ T. Kim, ${ }^{147}$ T. Kim, ${ }^{147}$ S. Kirsch, ${ }^{38,68}$ I. Kisel, ${ }^{38}$ S. Kiselev, ${ }^{91}$ A. Kisiel ${ }^{142}$ J. L. Klay, ${ }^{5}$ C. Klein ${ }^{68}$ J. Klein, ${ }^{58}$ S. Klein,${ }^{79}$ C. Klein-Bösing, ${ }^{144}$ M. Kleiner ${ }^{68}$ A. Kluge, ${ }^{33}$ M. L. Knichel, ${ }^{33}$ A. G. Knospe, ${ }^{125}$ C. Kobdaj, ${ }^{115}$ M. K. Köhler, ${ }^{103}$ T. Kollegger, ${ }^{106}$ A. Kondratyev, ${ }^{75}$ N. Kondratyeva, ${ }^{92}$ E. Kondratyuk, ${ }^{90}$ J. Konig, ${ }^{68}$ P. J. Konopka, ${ }^{33}$ L. Koska, ${ }^{116}$ O. Kovalenko, ${ }^{84}$ V. Kovalenko, ${ }^{112}$ M. Kowalski, ${ }^{118}$ I. Králik, ${ }^{64}$ A. Kravčáková, ${ }^{37}$

L. Kreis, ${ }^{106}$ M. Krivda, ${ }^{64,110}$ F. Krizek, ${ }^{94}$ K. Krizkova Gajdosova, ${ }^{36}$ M. Krüger ${ }^{68}$ E. Kryshen, ${ }^{97}$ M. Krzewicki, ${ }^{38}$ A. M. Kubera, ${ }^{96}$ V. Kučera,${ }^{60}$ C. Kuhn, ${ }^{136}$ P. G. Kuijer,${ }^{89}$ L. Kumar, ${ }^{99}$ S. Kumar, ${ }^{48}$ S. Kundu,${ }^{85}$ P. Kurashvili, ${ }^{84}$ A. Kurepin, ${ }^{62}$ A. B. Kurepin, ${ }^{62}$ A. Kuryakin, ${ }^{108}$ S. Kushpil,${ }^{94}$ J. Kvapil, ${ }^{110}$ M. J. Kweon, ${ }^{60}$ J. Y. Kwon, ${ }^{60}$ Y. Kwon, ${ }^{147}$ S. L. La Pointe, ${ }^{38}$ P. La Rocca, ${ }^{27}$ Y. S. Lai,${ }^{79}$ R. Langoy, ${ }^{129}$ K. Lapidus, ${ }^{33}$ A. Lardeux,${ }^{20}$ P. Larionov,${ }^{51}$ E. Laudi, ${ }^{33}$ R. Lavicka,${ }^{36}$ T. Lazareva, ${ }^{112}$ R. Lea, ${ }^{24}$ L. Leardini, ${ }^{103}$ J. Lee, ${ }^{133}$ S. Lee,${ }^{147}$ F. Lehas, ${ }^{89}$ S. Lehner, ${ }^{113}$ J. Lehrbach, ${ }^{38}$ R. C. Lemmon, ${ }^{93}$ I. León Monzón, ${ }^{120}$ E. D. Lesser, ${ }^{19}$ M. Lettrich, ${ }^{33}$ P. Lévai, ${ }^{145}$ X. Li, ${ }^{12}$ X. L. Li,${ }^{6}$ J. Lien, ${ }^{129}$ R. Lietava, ${ }^{110}$ B. Lim, ${ }^{17}$ V. Lindenstruth, ${ }^{38}$ S. W. Lindsay, ${ }^{127}$ C. Lippmann, ${ }^{106}$ M. A. Lisa,${ }^{96}$ V. Litichevskyi, ${ }^{43}$ A. Liu, ${ }^{19}$ S. Liu, ${ }^{96}$ W. J. Llope,${ }^{143}$ I. M. Lofnes ${ }^{21}$ V. Loginov, ${ }^{92}$ C. Loizides, ${ }^{95}$ P. Loncar, ${ }^{34}$ X. Lopez, ${ }^{134}$ E. López Torres, ${ }^{8}$ J. R. Luhder, ${ }^{144}$ M. Lunardon,${ }^{28}$ G. Luparello,${ }^{59}$ Y. Ma, ${ }^{39}$ A. Maevskaya, ${ }^{62}$ M. Mager ${ }^{33}$ S. M. Mahmood, ${ }^{20}$ T. Mahmoud, ${ }^{42}$ A. Maire, ${ }^{136}$ R. D. Majka, ${ }^{146}$ M. Malaev, ${ }^{97}$ Q. W. Malik ${ }^{20}$ L. Malinina,${ }^{75, \mathrm{c}}$ D. Mal'Kevich, ${ }^{91}$ P. Malzacher, ${ }^{106}$ G. Mandaglio, ${ }^{55}$ V. Manko,${ }^{87}$ F. Manso, ${ }^{134}$ V. Manzari,${ }^{52}$ Y. Mao, ${ }^{6}$ M. Marchisone, ${ }^{135}$ J. Mareš, ${ }^{66}$ G. V. Margagliotti, ${ }^{24}$ A. Margotti, ${ }^{53}$ J. Margutti, ${ }^{63}$ A. Marín, ${ }^{106}$ C. Markert, ${ }^{119}$

M. Marquard ${ }^{68}$ N. A. Martin, ${ }^{103}$ P. Martinengo, ${ }^{33}$ J.L. Martinez, ${ }^{125}$ M. I. Martínez, ${ }^{44}$ G. Martínez García, ${ }^{114}$

M. Martinez Pedreira, ${ }^{33}$ S. Masciocchi, ${ }^{106}$ M. Masera, ${ }^{25}$ A. Masoni, ${ }^{54}$ L. Massacrier, ${ }^{61}$ E. Masson, ${ }^{114}$ A. Mastroserio, ${ }^{52,138}$ A. M. Mathis ${ }^{104,117}$ O. Matonoha, ${ }^{80}$ P. F. T. Matuoka ${ }^{121}$ A. Matyja,${ }^{118}$ C. Mayer, ${ }^{118}$ M. Mazzilli, ${ }^{52}$ M. A. Mazzoni ${ }^{57}$ A. F. Mechler, ${ }^{68}$ F. Meddi, ${ }^{22}$ Y. Melikyan, ${ }^{62,92}$ A. Menchaca-Rocha, ${ }^{71}$ C. Mengke, ${ }^{6}$ E. Meninno,${ }^{29,113}$ M. Meres ${ }^{13}$ S. Mhlanga, ${ }^{124}$ Y. Miake, ${ }^{133}$ L. Micheletti, ${ }^{25}$ D. L. Mihaylov, ${ }^{104}$ K. Mikhaylov, ${ }^{75,91}$ A. Mischke, ${ }^{63, a}$ A. N. Mishra, ${ }^{69}$ D. Miśkowiec, ${ }^{106}$ A. Modak, ${ }^{3}$ N. Mohammadi, ${ }^{33}$ A. P. Mohanty, ${ }^{63}$ B. Mohanty, ${ }^{85}$ M. Mohisin Khan, ${ }^{16, d}$ C. Mordasini, ${ }^{104}$ D. A. Moreira De Godoy, ${ }^{144}$ L. A. P. Moreno, ${ }^{44}$ I. Morozov, ${ }^{62}$ A. Morsch, ${ }^{33}$ T. Mrnjavac, ${ }^{33}$ V. Muccifora, ${ }^{51}$ E. Mudnic ${ }^{34}$ D. Mühlheim, ${ }^{144}$ S. Muhuri, ${ }^{141}$ J. D. Mulligan, ${ }^{79}$ M. G. Munhoz, ${ }^{121}$ R. H. Munzer, ${ }^{68}$ H. Murakami, ${ }^{132}$ S. Murray, ${ }^{124}$ L. Musa, ${ }^{33}$ J. Musinsky, ${ }^{64}$ C. J. Myers, ${ }^{125}$ J. W. Myrcha, ${ }^{142}$ B. Naik,${ }^{48}$ R. Nair,${ }^{84}$ B. K. Nandi, ${ }^{48}$ R. Nania,${ }^{10,53}$ E. Nappi ${ }^{52}$ M. U. Naru, ${ }^{14}$ A. F. Nassirpour, ${ }^{80}$ C. Nattrass, ${ }^{130}$ R. Nayak, ${ }^{48}$ T. K. Nayak,${ }^{85}$ S. Nazarenko, ${ }^{108}$ A. Neagu, ${ }^{20}$

R. A. Negrao De Oliveira, ${ }^{68}$ L. Nellen, ${ }^{69}$ S. V. Nesbo, ${ }^{35}$ G. Neskovic, ${ }^{38}$ D. Nesterov, ${ }^{112}$ L. T. Neumann, ${ }^{142}$ B. S. Nielsen, ${ }^{88}$ S. Nikolaev ${ }^{87}$ S. Nikulin, ${ }^{87}$ V. Nikulin, ${ }^{97}$ F. Noferini,${ }^{10,53}$ P. Nomokonov,${ }^{75}$ J. Norman,${ }^{78,127}$ N. Novitzky, ${ }^{133}$

P. Nowakowski, ${ }^{142}$ A. Nyanin, ${ }^{87}$ J. Nystrand, ${ }^{21}$ M. Ogino, ${ }^{81}$ A. Ohlson,${ }^{80,103}$ J. Oleniacz, ${ }^{142}$ A. C. Oliveira Da Silva, ${ }^{121,130}$ M. H. Oliver, ${ }^{146}$ C. Oppedisano, ${ }^{58}$ R. Orava, ${ }^{43}$ A. Ortiz Velasquez,${ }^{69}$ A. Oskarsson, ${ }^{80}$ J. Otwinowski, ${ }^{118}$ K. Oyama, ${ }^{81}$ Y. Pachmayer, ${ }^{103}$ V. Pacik, ${ }^{88}$ D. Pagano, ${ }^{140}$ G. Paic,${ }^{69}$ J. Pan, ${ }^{143}$ A. K. Pandey ${ }^{48}$ S. Panebianco,${ }^{137}$ P. Pareek,${ }^{49,141}$ J. Park, ${ }^{60}$ J. E. Parkkila, ${ }^{126}$ S. Parmar, ${ }^{99}$ S. P. Pathak, ${ }^{125}$ R. N. Patra, ${ }^{141}$ B. Paul,${ }^{23,58}$ H. Pei,${ }^{6}$ T. Peitzmann, ${ }^{63}$ X. Peng, ${ }^{6}$ L. G. Pereira ${ }^{70}$ H. Pereira Da Costa, ${ }^{137}$ D. Peresunko, ${ }^{87}$ G. M. Perez, ${ }^{8}$ E. Perez Lezama ${ }^{68}$ V. Peskov,${ }^{68}$ Y. Pestov, ${ }^{4}$ V. Petráček, ${ }^{36}$ M. Petrovici, ${ }^{47}$ R. P. Pezzi, ${ }^{70}$ S. Piano, ${ }^{59}$ M. Pikna, ${ }^{13}$ P. Pillot,${ }^{114}$ O. Pinazza, ${ }^{33,53}$ L. Pinsky, ${ }^{125}$ C. Pinto, ${ }^{27}$ S. Pisano, ${ }^{10,51}$ 
D. Pistone,${ }^{55}$ M. Płoskoń, ${ }^{79}$ M. Planinic, ${ }^{98}$ F. Pliquett, ${ }^{68}$ J. Pluta, ${ }^{142}$ S. Pochybova, ${ }^{145, a}$ M. G. Poghosyan, ${ }^{95}$ B. Polichtchouk ${ }^{90}$ N. Poljak, ${ }^{98}$ A. Pop, ${ }^{47}$ H. Poppenborg, ${ }^{144}$ S. Porteboeuf-Houssais, ${ }^{134}$ V. Pozdniakov,${ }^{75}$ S. K. Prasad ${ }^{3}$ R. Preghenella, ${ }^{53}$ F. Prino, ${ }^{58}$ C. A. Pruneau, ${ }^{143}$ I. Pshenichnov, ${ }^{62}$ M. Puccio, ${ }^{25,33}$ J. Putschke, ${ }^{143}$ R. E. Quishpe, ${ }^{125}$ S. Ragoni, ${ }^{110}$ S. Raha, ${ }^{3}$ S. Rajput, ${ }^{100}$ J. Rak, ${ }^{126}$ A. Rakotozafindrabe, ${ }^{137}$ L. Ramello, ${ }^{31}$ F. Rami, ${ }^{136}$ R. Raniwala, ${ }^{101}$ S. Raniwala, ${ }^{101}$ S. S. Räsänen, ${ }^{43}$ R. Rath, ${ }^{49}$ V. Ratza, ${ }^{42}$ I. Ravasenga, ${ }^{30,89}$ K. F. Read, ${ }^{95,130}$ K. Redlich,${ }^{84, e}$ A. Rehman, ${ }^{21}$ P. Reichelt, ${ }^{68}$ F. Reidt, ${ }^{33}$ X. Ren, ${ }^{6}$ R. Renfordt, ${ }^{68}$ Z. Rescakova, ${ }^{37}$ J.-P. Revol, ${ }^{10}$ K. Reygers, ${ }^{103}$ V. Riabov, ${ }^{97}$ T. Richert,${ }^{80,88}$ M. Richter, ${ }^{20}$ P. Riedler, ${ }^{33}$ W. Riegler, ${ }^{33}$ F. Riggi, ${ }^{27}$ C. Ristea, ${ }^{67}$ S. P. Rode, ${ }^{49}$ M. Rodríguez Cahuantzi ${ }^{44}$ K. Røed, ${ }^{20}$ R. Rogalev, ${ }^{90}$ E. Rogochaya, ${ }^{75}$ D. Rohr,${ }^{33}$ D. Röhrich,${ }^{21}$ P. S. Rokita, ${ }^{142}$ F. Ronchetti, ${ }^{51}$ E. D. Rosas, ${ }^{69}$ K. Roslon, ${ }^{142}$ A. Rossi ${ }^{28,56}$ A. Rotondi, ${ }^{139}$ A. Roy, ${ }^{49}$ P. Roy, ${ }^{109}$ O. V. Rueda,${ }^{80}$ R. Rui,${ }^{24}$ B. Rumyantsev, ${ }^{75}$ A. Rustamov, ${ }^{86}$ E. Ryabinkin, ${ }^{87}$ Y. Ryabov, ${ }^{97}$ A. Rybicki, ${ }^{118}$ H. Rytkonen, ${ }^{126}$ O. A. M. Saarimaki, ${ }^{43}$ S. Sadhu, ${ }^{141}$ S. Sadovsky, ${ }^{90}$ K. Šafařík, ${ }^{36}$ S. K. Saha, ${ }^{141}$ B. Sahoo, ${ }^{48}$ P. Sahoo, ${ }^{48,49}$ R. Sahoo, ${ }^{49}$ S. Sahoo, ${ }^{65}$ P. K. Sahu, ${ }^{65}$ J. Saini, ${ }^{141}$ S. Sakai, ${ }^{133}$ S. Sambyal, ${ }^{100}$ V. Samsonov, ${ }^{92,97}$ D. Sarkar, ${ }^{143}$ N. Sarkar, ${ }^{141}$ P. Sarma, ${ }^{41}$ V. M. Sarti, ${ }^{104}$ M. H. P. Sas, ${ }^{63}$ E. Scapparone, ${ }^{53}$ B. Schaefer, ${ }^{95}$ J. Schambach, ${ }^{119}$ H. S. Scheid, ${ }^{68}$ C. Schiaua, ${ }^{47}$ R. Schicker, ${ }^{103}$ A. Schmah, ${ }^{103}$ C. Schmidt,${ }^{106}$ H. R. Schmidt, ${ }^{102}$ M. O. Schmidt, ${ }^{103}$ M. Schmidt, ${ }^{102}$ N. V. Schmidt, ${ }^{68,95}$ A. R. Schmier, ${ }^{130}$ J. Schukraft, ${ }^{88}$ Y. Schutz, ${ }^{33,136}$ K. Schwarz, ${ }^{106}$ K. Schweda, ${ }^{106}$ G. Scioli, ${ }^{26}$ E. Scomparin, ${ }^{58}$ M. Šefčík, ${ }^{37}$ J. E. Seger, ${ }^{15}$ Y. Sekiguchi, ${ }^{132}$ D. Sekihata, ${ }^{132}$ I. Selyuzhenkov, ${ }^{92,106}$ S. Senyukov, ${ }^{136}$ D. Serebryakov, ${ }^{62}$ E. Serradilla, ${ }^{71}$ A. Sevcenco, ${ }^{67}$ A. Shabanov, ${ }^{62}$ A. Shabetai, ${ }^{114}$ R. Shahoyan, ${ }^{33}$ W. Shaikh, ${ }^{109}$ A. Shangaraev, ${ }^{90}$ A. Sharma, ${ }^{99}$ A. Sharma, ${ }^{100}$ H. Sharma, ${ }^{118}$ M. Sharma, ${ }^{100}$ N. Sharma,${ }^{99}$ A. I. Sheikh, ${ }^{141}$ K. Shigaki, ${ }^{45}$ M. Shimomura, ${ }^{82}$ S. Shirinkin, ${ }^{91}$ Q. Shou, ${ }^{39}$ Y. Sibiriak,${ }^{87}$ S. Siddhanta, ${ }^{54}$ T. Siemiarczuk, ${ }^{84}$ D. Silvermyr,${ }^{80}$ G. Simatovic, ${ }^{89}$ G. Simonetti, ${ }^{33,104}$ R. Singh ${ }^{85}$ R. Singh, ${ }^{100}$ R. Singh, ${ }^{49}$ V. K. Singh, ${ }^{141}$ V. Singhal, ${ }^{141}$ T. Sinha, ${ }^{109}$ B. Sitar, ${ }^{13}$

M. Sitta ${ }^{31}$ T. B. Skaali, ${ }^{20}$ M. Slupecki, ${ }^{126}$ N. Smirnov, ${ }^{146}$ R. J. M. Snellings, ${ }^{63}$ T. W. Snellman, ${ }^{43,126}$ C. Soncco, ${ }^{111}$ J. Song, ${ }^{60,125}$ A. Songmoolnak, ${ }^{115}$ F. Soramel, ${ }^{28}$ S. Sorensen, ${ }^{130}$ I. Sputowska, ${ }^{118}$ J. Stachel, ${ }^{103}$ I. Stan, ${ }^{67}$ P. Stankus, ${ }^{95}$ P. J. Steffanic, ${ }^{130}$ E. Stenlund, ${ }^{80}$ D. Stocco,${ }^{114}$ M. M. Storetvedt, ${ }^{35}$ L. D. Stritto, ${ }^{29}$ A. A. P. Suaide, ${ }^{121}$ T. Sugitate, ${ }^{45}$ C. Suire, ${ }^{61}$ M. Suleymanov, ${ }^{14}$ M. Suljic, ${ }^{33}$ R. Sultanov,${ }^{91}$ M. Šumbera, ${ }^{94}$ S. Sumowidagdo, ${ }^{50}$ S. Swain, ${ }^{65}$ A. Szabo, ${ }^{13}$ I. Szarka, ${ }^{13}$ U. Tabassam, ${ }^{14}$ G. Taillepied, ${ }^{134}$ J. Takahashi, ${ }^{122}$ G. J. Tambave, ${ }^{21}$ S. Tang ${ }^{6,134}$ M. Tarhini, ${ }^{114}$ M. G. Tarzila ${ }^{47}$ A. Tauro, ${ }^{33}$ G. Tejeda Muñoz, ${ }^{44}$ A. Telesca ${ }^{33}$ C. Terrevoli, ${ }^{125}$ D. Thakur, ${ }^{49}$ S. Thakur, ${ }^{141}$ D. Thomas, ${ }^{119}$ F. Thoresen,${ }^{88}$ R. Tieulent, ${ }^{135}$ A. Tikhonov ${ }^{62}$ A. R. Timmins, ${ }^{125}$ A. Toia,${ }^{68}$ N. Topilskaya, ${ }^{62}$ M. Toppi, ${ }^{51}$ F. Torales-Acosta, ${ }^{19}$ S. R. Torres, ${ }^{9,120}$ A. Trifiro, ${ }^{55}$ S. Tripathy, ${ }^{49}$ T. Tripathy, ${ }^{48}$ S. Trogolo, ${ }^{28}$ G. Trombetta, ${ }^{32}$ L. Tropp,${ }^{37}$ V. Trubnikov, ${ }^{2}$ W. H. Trzaska, ${ }^{126}$ T. P. Trzcinski, ${ }^{142}$ B. A. Trzeciak, ${ }^{63}$ T. Tsuji, ${ }^{132}$ A. Tumkin, ${ }^{108}$ R. Turrisi, ${ }^{56}$ T. S. Tveter ${ }^{20}$ K. Ullaland, ${ }^{21}$ E. N. Umaka, ${ }^{125}$ A. Uras, ${ }^{135}$

G. L. Usai, ${ }^{23}$ A. Utrobicic, ${ }^{98}$ M. Vala, ${ }^{37}$ N. Valle, ${ }^{139}$ S. Vallero, ${ }^{58}$ N. van der Kolk, ${ }^{63}$ L. V. R. van Doremalen, ${ }^{63}$

M. van Leeuwen, ${ }^{63}$ P. Vande Vyvre, ${ }^{33}$ D. Varga, ${ }^{145}$ Z. Varga,${ }^{145}$ M. Varga-Kofarago, ${ }^{145}$ A. Vargas,${ }^{44}$ M. Vasileiou, ${ }^{83}$

A. Vasiliev, ${ }^{87}$ O. Vázquez Doce, ${ }^{104,117}$ V. Vechernin, ${ }^{112}$ A. M. Veen ${ }^{63}$ E. Vercellin, ${ }^{25}$ S. Vergara Limón, ${ }^{44}$ L. Vermunt,${ }^{63}$

R. Vernet, ${ }^{7}$ R. Vértesi, ${ }^{145}$ L. Vickovic, ${ }^{34}$ Z. Vilakazi, ${ }^{131}$ O. Villalobos Baillie, ${ }^{110}$ A. Villatoro Tello, ${ }^{44}$ G. Vino, ${ }^{52}$

A. Vinogradov, ${ }^{87}$ T. Virgili, ${ }^{29}$ V. Vislavicius, ${ }^{88}$ A. Vodopyanov, ${ }^{75}$ B. Volkel,${ }^{33}$ M. A. Völkl, ${ }^{102}$ K. Voloshin, ${ }^{91}$ S. A. Voloshin, ${ }^{143}$ G. Volpe, ${ }^{32}$ B. von Haller, ${ }^{33}$ I. Vorobyev, ${ }^{104}$ D. Voscek, ${ }^{116}$ J. Vrláková, ${ }^{37}$ B. Wagner, ${ }^{21}$ M. Weber, ${ }^{113}$ S. G. Weber, ${ }^{144}$ A. Wegrzynek, ${ }^{33}$ D. F. Weiser, ${ }^{103}$ S. C. Wenzel, ${ }^{33}$ J. P. Wessels, ${ }^{144}$ J. Wiechula, ${ }^{68}$ J. Wikne, ${ }^{20}$ G. Wilk, ${ }^{84}$ J. Wilkinson, ${ }^{10,53}$ G. A. Willems, ${ }^{33}$ E. Willsher, ${ }^{110}$ B. Windelband, ${ }^{103}$ M. Winn, ${ }^{137}$ W. E. Witt, ${ }^{130}$ Y. Wu, ${ }^{128}$ R. Xu, ${ }^{6}$

S. Yalcin, ${ }^{77}$ K. Yamakawa, ${ }^{45}$ S. Yang, ${ }^{21}$ S. Yano, ${ }^{137}$ Z. Yin, ${ }^{6}$ H. Yokoyama ${ }^{63}$ I.-K. Yoo, ${ }^{17}$ J. H. Yoon, ${ }^{60}$ S. Yuan, ${ }^{21}$ A. Yuncu, ${ }^{103}$ V. Yurchenko, ${ }^{2}$ V. Zaccolo, ${ }^{24}$ A. Zaman, ${ }^{14}$ C. Zampolli, ${ }^{33}$ H. J. C. Zanoli, ${ }^{63}$ N. Zardoshti, ${ }^{33}$ A. Zarochentsev, ${ }^{112}$ P. Závada, ${ }^{66}$ N. Zaviyalov, ${ }^{108}$ H. Zbroszczyk, ${ }^{142}$ M. Zhalov, ${ }^{97}$ S. Zhang, ${ }^{39}$ X. Zhang, ${ }^{6}$ Z. Zhang, ${ }^{6}$ V. Zherebchevskii, ${ }^{112}$ D. Zhou, ${ }^{6}$ Y. Zhou, ${ }^{88}$ Z. Zhou, ${ }^{21}$ J. Zhu, ${ }^{6,106}$ Y. Zhu, ${ }^{6}$ A. Zichichi, ${ }^{10,26}$ M. B. Zimmermann, ${ }^{33}$ G. Zinovjev, ${ }^{2}$ and N. Zurlo ${ }^{140}$

(The ALICE Collaboration)

\footnotetext{
${ }^{1}$ A.I. Alikhanyan National Science Laboratory (Yerevan Physics Institute) Foundation, Yerevan, Armenia

${ }^{2}$ Bogolyubov Institute for Theoretical Physics, National Academy of Sciences of Ukraine, Kiev, Ukraine

${ }^{3}$ Bose Institute, Department of Physics and Centre for Astroparticle Physics and Space Science (CAPSS), Kolkata, India

${ }^{4}$ Budker Institute for Nuclear Physics, Novosibirsk, Russia

${ }^{5}$ California Polytechnic State University, San Luis Obispo, California, United States

${ }^{6}$ Central China Normal University, Wuhan, China
} 
${ }^{7}$ Centre de Calcul de l'IN2P3, Villeurbanne, Lyon, France

${ }^{8}$ Centro de Aplicaciones Tecnológicas y Desarrollo Nuclear (CEADEN), Havana, Cuba

${ }^{9}$ Centro de Investigación y de Estudios Avanzados (CINVESTAV), Mexico City and Mérida, Mexico

${ }^{10}$ Centro Fermi-Museo Storico della Fisica e Centro Studi e Ricerche "Enrico Fermi," Rome, Italy

${ }^{11}$ Chicago State University, Chicago, Illinois, United States

${ }^{12}$ China Institute of Atomic Energy, Beijing, China

${ }^{13}$ Comenius University Bratislava, Faculty of Mathematics, Physics and Informatics, Bratislava, Slovakia

${ }^{14}$ COMSATS University Islamabad, Islamabad, Pakistan

${ }^{15}$ Creighton University, Omaha, Nebraska, United States

${ }^{16}$ Department of Physics, Aligarh Muslim University, Aligarh, India

${ }^{17}$ Department of Physics, Pusan National University, Pusan, Republic of Korea

${ }^{18}$ Department of Physics, Sejong University, Seoul, Republic of Korea

${ }^{19}$ Department of Physics, University of California, Berkeley, California, United States

${ }^{20}$ Department of Physics, University of Oslo, Oslo, Norway

${ }^{21}$ Department of Physics and Technology, University of Bergen, Bergen, Norway

${ }^{22}$ Dipartimento di Fisica dell'Università “La Sapienza” and Sezione INFN, Rome, Italy

${ }^{23}$ Dipartimento di Fisica dell'Università and Sezione INFN, Cagliari, Italy

${ }^{24}$ Dipartimento di Fisica dell'Università and Sezione INFN, Trieste, Italy

${ }^{25}$ Dipartimento di Fisica dell'Università and Sezione INFN, Turin, Italy

${ }^{26}$ Dipartimento di Fisica e Astronomia dell'Università and Sezione INFN, Bologna, Italy

${ }^{27}$ Dipartimento di Fisica e Astronomia dell'Università and Sezione INFN, Catania, Italy

${ }^{28}$ Dipartimento di Fisica e Astronomia dell'Università and Sezione INFN, Padova, Italy

${ }^{29}$ Dipartimento di Fisica "E.R. Caianiello" dell'Università and Gruppo Collegato INFN, Salerno, Italy

${ }^{30}$ Dipartimento DISAT del Politecnico and Sezione INFN, Turin, Italy

${ }^{31}$ Dipartimento di Scienze e Innovazione Tecnologica dell'Università del Piemonte Orientale and INFN Sezione di Torino, Alessandria, Italy

${ }^{32}$ Dipartimento Interateneo di Fisica "M. Merlin" and Sezione INFN, Bari, Italy

${ }^{33}$ European Organization for Nuclear Research (CERN), Geneva, Switzerland

${ }^{34}$ Faculty of Electrical Engineering, Mechanical Engineering and Naval Architecture, University of Split, Split, Croatia

${ }^{35}$ Faculty of Engineering and Science, Western Norway University of Applied Sciences, Bergen, Norway

${ }^{36}$ Faculty of Nuclear Sciences and Physical Engineering, Czech Technical University in Prague, Prague, Czech Republic

${ }^{37}$ Faculty of Science, P.J. Šafárik University, Košice, Slovakia

${ }^{38}$ Frankfurt Institute for Advanced Studies, Johann Wolfgang Goethe-Universität Frankfurt, Frankfurt, Germany

${ }^{39}$ Fudan University, Shanghai, China

${ }^{40}$ Gangneung-Wonju National University, Gangneung, Republic of Korea

${ }^{41}$ Gauhati University, Department of Physics, Guwahati, India

${ }^{42}$ Helmholtz-Institut für Strahlen- und Kernphysik, Rheinische Friedrich-Wilhelms-Universität Bonn, Bonn, Germany

${ }^{43}$ Helsinki Institute of Physics (HIP), Helsinki, Finland

${ }^{44}$ High Energy Physics Group, Universidad Autónoma de Puebla, Puebla, Mexico

${ }^{45}$ Hiroshima University, Hiroshima, Japan

${ }^{46}$ Hochschule Worms, Zentrum für Technologietransfer und Telekommunikation (ZTT), Worms, Germany

${ }^{47}$ Horia Hulubei National Institute of Physics and Nuclear Engineering, Bucharest, Romania

${ }^{48}$ Indian Institute of Technology Bombay (IIT), Mumbai, India

${ }^{49}$ Indian Institute of Technology Indore, Indore, India

${ }^{50}$ Indonesian Institute of Sciences, Jakarta, Indonesia

${ }^{51}$ INFN, Laboratori Nazionali di Frascati, Frascati, Italy

${ }^{52}$ INFN, Sezione di Bari, Bari, Italy

${ }^{53}$ INFN, Sezione di Bologna, Bologna, Italy

${ }^{54}$ INFN, Sezione di Cagliari, Cagliari, Italy

${ }^{55}$ INFN, Sezione di Catania, Catania, Italy

${ }^{56}$ INFN, Sezione di Padova, Padova, Italy

${ }^{57}$ INFN, Sezione di Roma, Rome, Italy

${ }^{58}$ INFN, Sezione di Torino, Turin, Italy

${ }^{59}$ INFN, Sezione di Trieste, Trieste, Italy

${ }^{60}$ Inha University, Incheon, Republic of Korea

${ }^{61}$ Institut de Physique Nucléaire d'Orsay (IPNO), Institut National de Physique Nucléaire et de Physique des Particules (IN2P3/CNRS),

Université de Paris-Sud, Université Paris-Saclay, Orsay, France

${ }^{62}$ Institute for Nuclear Research, Academy of Sciences, Moscow, Russia

${ }^{63}$ Institute for Subatomic Physics, Utrecht University/Nikhef, Utrecht, Netherlands

${ }^{64}$ Institute of Experimental Physics, Slovak Academy of Sciences, Košice, Slovakia 


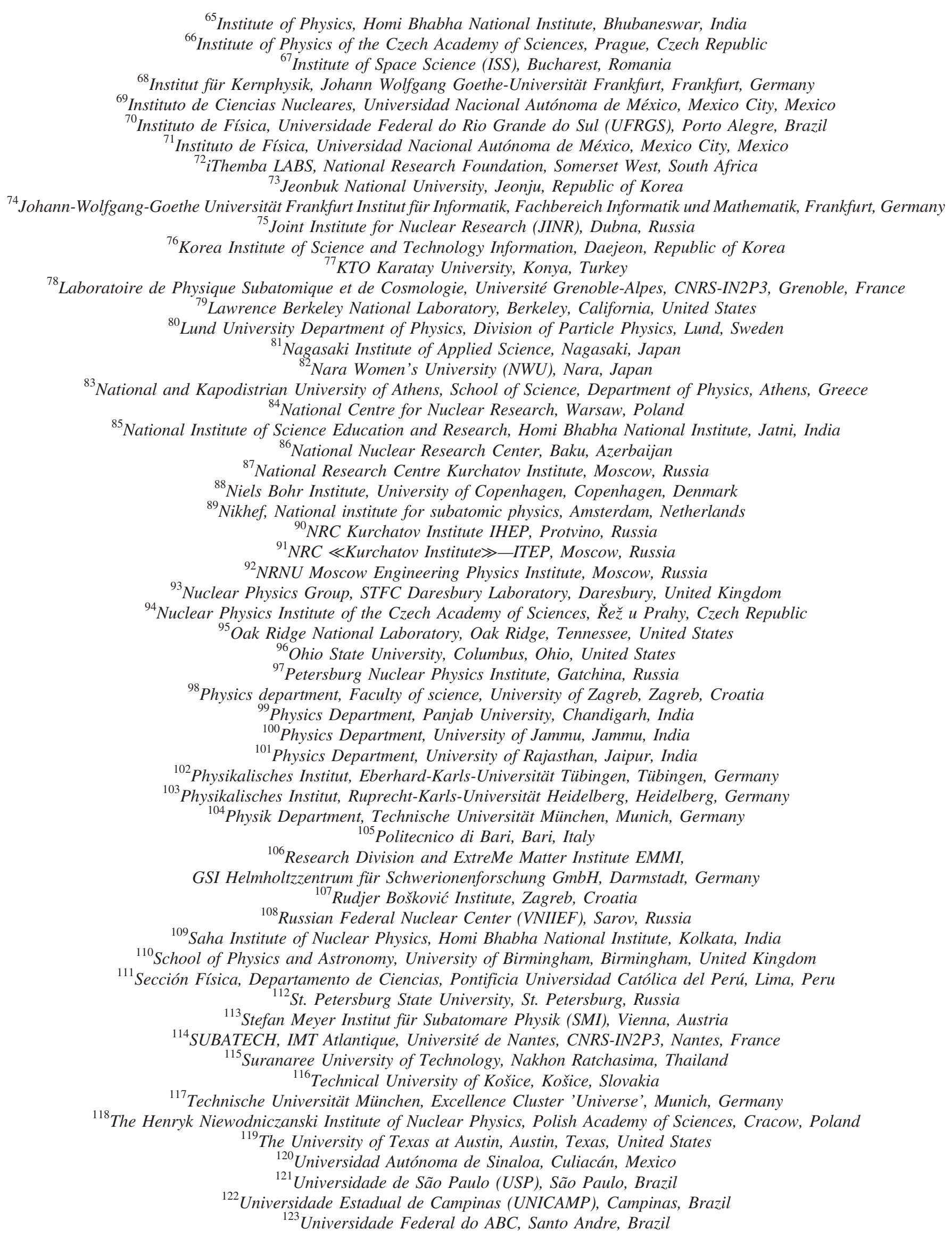


${ }^{124}$ University of Cape Town, Cape Town, South Africa

${ }^{125}$ University of Houston, Houston, Texas, United States

${ }^{126}$ University of Jyväskylä, Jyväskylä, Finland

${ }^{127}$ University of Liverpool, Liverpool, United Kingdom

${ }^{128}$ University of Science and Technology of China, Hefei, China

${ }^{129}$ University of South-Eastern Norway, Tonsberg, Norway

${ }^{130}$ University of Tennessee, Knoxville, Tennessee, United States

${ }^{131}$ University of the Witwatersrand, Johannesburg, South Africa

${ }^{132}$ University of Tokyo, Tokyo, Japan

${ }^{133}$ University of Tsukuba, Tsukuba, Japan

${ }^{134}$ Université Clermont Auvergne, CNRS/IN2P3, LPC, Clermont-Ferrand, France

${ }^{135}$ Université de Lyon, Université Lyon 1, CNRS/IN2P3, IPN-Lyon, Villeurbanne, Lyon, France

${ }^{136}$ Université de Strasbourg, CNRS, IPHC UMR 7178, F-67000 Strasbourg, France, Strasbourg, France

${ }^{137}$ Université Paris-Saclay Centre d'Etudes de Saclay (CEA), IRFU, Départment de Physique Nucléaire (DPhN), Saclay, France

${ }^{138}$ Università degli Studi di Foggia, Foggia, Italy

${ }^{139}$ Università degli Studi di Pavia, Pavia, Italy

${ }^{140}$ Università di Brescia, Brescia, Italy

${ }^{141}$ Variable Energy Cyclotron Centre, Homi Bhabha National Institute, Kolkata, India

${ }^{142}$ Warsaw University of Technology, Warsaw, Poland

${ }^{143}$ Wayne State University, Detroit, Michigan, United States

${ }^{144}$ Westfälische Wilhelms-Universität Münster, Institut für Kernphysik, Münster, Germany

${ }^{145}$ Wigner Research Centre for Physics, Budapest, Hungary

${ }^{146}$ Yale University, New Haven, Connecticut, United States

${ }^{147}$ Yonsei University, Seoul, Republic of Korea

${ }^{\mathrm{a}}$ Deceased.

${ }^{\mathrm{b}}$ Dipartimento DET del Politecnico di Torino, Turin, Italy.

${ }^{\mathrm{c}}$ M.V. Lomonosov Moscow State University, D.V. Skobeltsyn Institute of Nuclear, Physics, Moscow, Russia.

${ }^{\mathrm{d} D}$ Department of Applied Physics, Aligarh Muslim University, Aligarh, India.

${ }^{\mathrm{e}}$ Institute of Theoretical Physics, University of Wroclaw, Poland. 